\title{
Rigorous lower bound on the scattering amplitude at large angles
}

\author{
Henri Epstein ${ }^{1}$ and André Martin ${ }^{2}$ \\ ${ }^{1}$ IHES, Bures-sur-Yvette, France \\ ${ }^{2}$ CERN, Geneva, Switzerland
}

(Received 13 March 2019; published 26 June 2019)

\begin{abstract}
We prove a lower bound for the modulus of the amplitude for a two-body process at large scattering angles. This is based on the interplay of the analyticity of the amplitude and the positivity properties of its absorptive part. The assumptions are minimal, namely, those of local quantum field theory (in the case when dispersion relations hold). In the Appendix A, lower bounds for the forward particle-particle and particle-antiparticle amplitudes are obtained. This is of independent interest.
\end{abstract}

DOI: 10.1103/PhysRevD.99.114025

\section{INTRODUCTION}

In 1963, Cérulus and one of us (A.M.) obtained a lower bound on the scattering amplitude at large angles [1]. It is not exactly a lower bound at a given angle because it is impossible to prevent the scattering amplitude from vanishing at a given point. What we obtained is a lower bound on the maximum of the modulus of the amplitude in some finite angular interval. The assumption made was the validity of the Mandelstam representation with a finite number of subtractions [2]. Another assumption was that the forward scattering amplitude cannot decrease faster than a power of $s$, the square of the c.m. energy. However, this assumption was removed by Jin and Martin in 1964 [3] since, at least for $\pi \pi, p \pi, K \pi$ scattering, the forward amplitude cannot decrease faster than $1 / s^{2}$ because of the positivity of the absorptive part in the forward direction. More exactly, there is at least a sequence of energies going to infinity for which this is true. The lower bound was

$$
C \exp (-\sqrt{s} \ln s)
$$

where $s$ is the square of the center-of-mass energy. At the time, it was a rather good surprise because experiments done by the Cornell group indicated that the large angle protonproton scattering amplitude behaved like $C \exp (-\sqrt{s})$ [4]. Thus, we disagreed with experiment only by a factor $\ln s$ in the exponential.

It so happens that this lower bound is violated by the Veneziano amplitude [5]. This is not astonishing because the Veneziano amplitude implies linearly rising Regge

Published by the American Physical Society under the terms of the Creative Commons Attribution 4.0 International license. Further distribution of this work must maintain attribution to the author(s) and the published article's title, journal citation, and DOI. Funded by SCOAP ${ }^{3}$. trajectories which means an infinite number of subtractions. Thus, Veneziano asked if there exists a "rigorous lower bound" (in the sense previously given) on the large angle scattering amplitude. After the work of one of us on the enlargement of the domain of analyticity in local field theory by using positivity properties of the absorptive part [6] and also the obtention of a lower bound of the forward absorptive part [7], this turned out to be possible, but the problem was left unsolved for about 50 years. In the present work we give an answer, probably the best possible one, but this answer is unfortunately an extremely small lower bound; thus, we publish our results rather as a matter of principle.

The strategy is the following:

(1) From [6] the absorptive part of the scattering amplitude is analytic in an ellipse in the $\cos \theta$ variable with foci at \pm 1 and a right extremity at $\cos \theta=1+2 m_{\pi}^{2} / k^{2}, k$ being the c.m. momentum. The modulus of the absorptive part is maximum at the right extremity of the ellipse, and morally the absorptive part is bounded by $s^{2}$ in the ellipse. Morally means that we only know from [6] that the integral of the absorptive part, divided by $s^{3}$, over $s$, is convergent for fixed momentum transfer, $t=$ $4 m_{\pi}^{2}-\varepsilon, \varepsilon>0$ arbitrarily small. In the special case of $\pi \pi$ scattering this integral is completely under control from the absolute bounds obtained previously ([8-10]).

(2) As we said before, the forward scattering amplitude has a moral lower bound $1 / s^{2}$; since the diffraction peak cannot be arbitrarily small because of the size of the ellipse, the elastic cross.section has a lower bound, and hence the forward absorptive part has a lower bound which is $1 / s^{5}(\log s)^{2}$ [7].

(3) If we have an upper bound on some angular interval of $-a<\cos \theta<+a$ and also an upper bound on the border of the ellipse, we can interpolate between these two bounds because the logarithm of the 
modulus of the absorptive part is a subharmonic function $($ of $\cos \theta)$, and we can get an upper bound anywhere inside the ellipse, in particular, at $\cos \theta=1$, i.e., the forward direction. If the postulated bound on the interval $(-a,+a)$ is too low, we will get a contradiction with the lower bound that we have in the forward direction. So the postulated upper bound on $(-a,+a)$ cannot be arbitrarily small.

However, things are not as simple as that because the lower bound on the scattering amplitude is only for discrete values of the energy; also, even if we assume that everything is continuous, we do not know if, precisely, for these discrete values, the absorptive part in the ellipse is bounded by $s^{2}$. To overcome this problem we replace the scattering amplitude by an average over some energy interval. The average has all the nice properties we want, but, as we shall see, we lose 1 power of $s$ in the bound on the absorptive part; however, this is unimportant. The next section is devoted to this averaging. The following section explains the interpolation described in (3) and gives the results. Details are given in the appendixes.

\section{NECESSITY OF AN AVERAGING OF THE SCATTERING AMPLITUDE ON THE ENERGY}

As stressed by Common [11] and Yndurain [12], we only know from [6] that the integral over the absorptive part $A_{s}$,

$$
\int_{\left(M_{1}+M_{2}\right)^{2}}^{\infty} \frac{A_{s}\left(s, t=4 m_{\pi}^{2}-\varepsilon\right)}{s^{3}} d s,
$$

is convergent. This does not mean that $A_{s}$ is less than $s^{2}$. In fact $A_{s}$ can be very large or even infinite for isolated values of $s$.

Since the forward scattering has a positive imaginary part for $s>0$ and a negative imaginary part for $s<0$, it has been shown [3], for a crossing-symmetric amplitude, that $\lim \sup s^{2}|F(s, 0)|>0$. This means that there is a sequence of values of $s,\left\{s_{i}\right\}$, going to infinity, for which $\lim _{i \rightarrow+\infty} s_{i}^{2} \mid F\left(s_{i}, 0\right)>0$. In fact, in all nonreal directions, $\lim s^{2}|F(s, 0)|>0$. For non-crossing-symmetric amplitudes, such as $\pi^{+} p \rightarrow \pi^{+} p$ and $\pi^{-} p \rightarrow \pi^{-} p$, we show in the present paper that $\lim s^{2+\epsilon}|F(s, 0)|>0, \epsilon>0$ arbitrarily small, both for $s \rightarrow+\infty$ and $s \rightarrow-\infty$ (i.e., $u \rightarrow+\infty)$. This will be proved in Appendix A. Thus, our results also hold for a nonsymmetric amplitude. The presence of $\epsilon$ is inessential.

Concerning the absorptive part, we have, from the optical theorem, $A_{s}(s, 0)>s \sigma_{\text {total }}>s \sigma_{\text {elastic }}$, and we need a lower bound on $\sigma_{\text {elastic }}$. Assume provisionally that, for $t=4 m_{\pi}^{2}-\varepsilon$, the absorptive part is bounded by $s^{N}$. We know that we cannot really assume that, but it will be corrected later by averaging. Then, using Schwarz's inequality, we have

$$
\begin{aligned}
& \left|\sum_{l=0}^{\infty}(2 l+1) f_{l}\right| \leq\left|\sum_{l=0}^{L}(2 l+1) f_{l}\right|+\left|\sum_{l=L+1}^{\infty}(2 l+1) f_{l}\right| \\
& \leq \sqrt{\left(\sum_{l=0}^{L}(2 l+1)\left|f_{l}\right|^{2}\right)} \times(L+1)+\left|\sum_{l=L+1}^{\infty}(2 l+1) f_{l}\right| \\
& \leq \sqrt{\left(\sum_{l=0}^{L}(2 l+1) \operatorname{Im} f_{l}\right)} \times(L+1)+\left|\sum_{l=L+1}^{\infty}(2 l+1) f_{l}\right|,
\end{aligned}
$$

where $L$ will be chosen later. If the absorptive part is bounded by $s^{N}$ for $T<4 m_{\pi}^{2}$,

$$
\left|\sum(2 l+1) \operatorname{Im} f_{l} P_{l}\left(1+\frac{T}{2 k^{2}}\right)\right|<s^{N},
$$

so by Schwarz's inequality

$$
\left|\sum_{L+1}^{\infty}(2 l+1) f_{l}\right|^{2} \leq \sum_{L+1}^{\infty} \frac{2 l+1}{P_{l}\left(1+\frac{T}{2 k^{2}}\right)} \sum_{0}^{\infty}(2 l+1) P_{l} \operatorname{Im} f_{l} .
$$

The second factor in (2.4) is bounded by $s^{N}$. The first factor can be calculated by using the inequality

$$
P_{l}(x)>\frac{1}{3}\left(x+\frac{1}{2} \sqrt{x^{2}-1}\right)^{l} \quad \text { for } x>1
$$

which follows from the integral representation of Legendre polynomials. We get, choosing $L=P \sqrt{s} \log s$, an asymptotic upper bound

$$
s^{-P \frac{\sqrt{\mathrm{T}}}{2 \sqrt{2}}} \times \frac{3 P s \log s}{\sqrt{\mathrm{T}}} .
$$

Choosing $P$ large enough compared to $N$, the second term in (2.2) is negligible, and we get

$$
|F(s, 0)|^{2}<P^{2} s(\log s)^{2} A_{s}(s, 0) .
$$

If

$$
|F(s, 0)|>\frac{1}{s^{2 \pm \varepsilon}}
$$

we get

$$
A_{s}>C \frac{1}{s^{5 \pm 2 \varepsilon}(\log s)^{2}} .
$$


Unfortunately we cannot assume $A_{s}(s, T)<s^{N}$, but this will be remedied now by averaging the amplitude over an energy interval.

To solve the problem we propose making an average over energies. We define

$$
\begin{aligned}
f(s, \cos \theta) & =F(s, t, u), \\
\cos \theta & =1+\frac{t}{2 k^{2}} .
\end{aligned}
$$

Now we average $f$ over an energy interval $\Delta$ :

$$
f_{\Delta}(s, \cos \theta)=\frac{1}{\Delta} \int_{s-\Delta}^{s} f\left(s^{\prime}, \cos \theta\right) d s^{\prime} .
$$

This averaging is mainly interesting for $s$ physical. However, in the special case of $\cos \theta=1$, it remains meaningful for $s<0$, i.e., for the $u$ channel. We take $\Delta$ smaller than the interval between the left cut and the right cut, and it follows that

$$
\lim _{s \rightarrow+\infty} \sup _{s}\left|f_{\Delta}(s, 1)\right| s^{2+\varepsilon}>0
$$

as in the case of $f$.

The reason why we take this averaging is that unitarity of the partial waves survives:

$$
f_{\Delta l}(s)=\frac{1}{\Delta} \int_{s-\Delta}^{s} f_{l}\left(s^{\prime}\right) d s^{\prime} .
$$

Now

$$
\operatorname{Im} f_{\Delta l}(s)=\frac{1}{\Delta} \int_{s-\Delta}^{s} \operatorname{Im} f_{l}\left(s^{\prime}\right) d s^{\prime}>\frac{1}{\Delta} \int_{s-\Delta}^{s}\left|f_{l}\left(s^{\prime}\right)\right|^{2} d s^{\prime} .
$$

But by Schwarz's inequality

$$
\frac{1}{\Delta} \int_{s-\Delta}^{s}\left|f_{l}\left(s^{\prime}\right)\right|^{2} d s^{\prime} \geq \frac{1}{\Delta^{2}}\left|\int_{s-\Delta}^{s}\right| f_{l}\left(s^{\prime}\right)\left|d s^{\prime}\right|^{2},
$$

so

$$
\operatorname{Im} f_{\Delta l}(s)>\left|f_{\Delta l}(s)\right|^{2} .
$$

The absorptive part is then averaged as

$$
\begin{gathered}
a_{\Delta}(s, \cos \theta)=\sum_{l=0}^{\infty} \operatorname{Im} f_{\Delta l}(s) P_{l}(\cos \theta), \\
a_{\Delta}(s, \cos \theta)=\frac{1}{\Delta} \int_{s-\Delta}^{s} A_{s}\left(s^{\prime}, t=(\cos \theta-1) 2 k^{\prime 2}\right) d s^{\prime} .
\end{gathered}
$$

For $\cos \theta>1$ we see that in the integrand $A_{s}\left(s^{\prime}, t=\right.$ $\left.(\cos \theta-1) 2 k^{\prime 2}\right)$ is less than $A_{s}\left(s^{\prime}, t=(\cos \theta-1) 2 k^{2}\right)$ since, in the interval $0 \leq t<4 m_{\pi}^{2}, A_{s}$ increases because the Legendre polynomial expansion converges.

Now remember that, according to [6],

$\int_{\left(M_{A}+M_{B}\right)^{2}}^{\infty} \frac{A_{s}\left(s^{\prime}, t\right)}{s^{\prime 3}} d s^{\prime} \quad$ converges for $t<4 m_{\pi}^{2}$.

Hence, for fixed $s \geq\left(M_{A}+M_{B}\right)^{2}+\Delta$ and $t<4 m_{\pi}^{2}$,

$$
\begin{aligned}
& a_{\Delta}\left(s, \cos \theta=1+\frac{t}{2 k^{2}}\right) \leq \frac{1}{\Delta} \int_{s-\Delta}^{s} A_{s}\left(s^{\prime}, t\right) d s^{\prime} \\
& \quad \leq \frac{s^{3}}{\Delta} \int_{\left(M_{A}+M_{B}\right)^{2}}^{\infty} \frac{A_{s}\left(s^{\prime}, t\right)}{s^{\prime 3}} d s^{\prime} .
\end{aligned}
$$

We conclude that

$$
a_{\Delta}\left(s, \cos \theta=1+\frac{t}{2 k^{2}}\right)<C s^{3} \quad \text { for } t<4 m_{\pi}^{2} .
$$

Thus, the previous argument, applied to $A_{s}$ with the assumption $A(s, t)<s^{N}$ for $t<4 m_{\pi}^{2}$, applies also to $a_{\Delta}$ with $N=3$. We realize that we are losing one power of $s$, but this is unimportant. We conclude that

$$
a_{\Delta}\left(s_{i}, \cos \theta=1\right)>\frac{C}{s_{i}^{5+2 \varepsilon}\left(\log s_{i}\right)^{2}}
$$

where $s_{i}$ belongs to the sequence where $\mid f_{\Delta}(s, \cos \theta=$ 1) $\mid s^{2+\varepsilon}$ approaches infinity.

\section{THE LEAST UPPER BOUND FOR $|\cos \theta| \leq a$}

Now we have all the ingredients to find a least upper bound of the scattering amplitude in an angular interval $|\cos \theta| \leq a$, for instance, $|\cos \theta| \leq \frac{1}{2}$ :

$$
a_{\Delta}\left(s_{i}, \cos \theta=1\right)>\frac{C}{s_{i}^{5+2 \varepsilon}\left(\log s_{i}\right)^{2}},
$$

$$
a_{\Delta}\left(s, \cos \theta=\frac{4 m_{\pi}^{2}-\eta}{2 k^{2}}\right)<C s^{3} .
$$

This bound, because of the positivity of the $\operatorname{Im} f_{\Delta l}$ 's, holds in the whole ellipse with foci at $\cos \theta= \pm 1$ and extremity at $\cos \theta=1+\frac{4 m_{\pi}^{2}-\eta}{2 k^{2}}$, with $\eta$ positive arbitrarily small.

To interpolate between the bounds (3.1) and (3.2) we use the following fact (proved in Appendix B): let $f$ be a function that is holomorphic in the domain $D_{L}$ bounded by the ellipse $E_{L}$ with foci \pm 1 and semi-major axis $\operatorname{ch}(L)$, $L>0$, 


$$
\begin{aligned}
& E_{L}=\{z: z=\cos (\theta+i L), \theta \in \mathbf{R}\} \\
& D_{L}=\{z: z=\cos (\theta+i y), \theta \in \mathbf{R}, y \in \mathbf{R},|y|<L\} .
\end{aligned}
$$

We suppose that $|f| \leq M$ on $D_{L}$ and $|f(z)| \leq m \forall z \in$ $[-a, a]$, where $0<m<M$ and $a=\cos (b), 0<b<\pi / 2$. Then

$$
|f(1)|<M^{1-\alpha} m^{\alpha},
$$

where, for very small $L$,

$$
\alpha=\frac{4}{\pi} \exp \left(\frac{-\pi b}{2 L}\right)
$$

[ $\alpha$ is the quantity denoted $1-H(1)$ in Appendix B]. We can rewrite (3.4) in the form

$$
m \geq M\left(\frac{|f(1)|}{M}\right)^{\frac{1}{\alpha}}
$$

We apply this to the case when $z=\cos (\theta)$, $f(z)=a_{\Delta}(s, \cos (\theta))$, and $s$ belongs to a certain real sequence tending to infinity such that (3.1) holds. We suppose, for simplicity, that all masses are equal to 1. We choose $a=\frac{1}{2} \Rightarrow b=\pi / 3$. The semi-major axis of the ellipse is $\operatorname{ch}(L)$,

$$
\begin{aligned}
\operatorname{ch}(L) & =1+\frac{4}{2 k^{2}}, \quad k^{2}=\frac{s}{4}-1, \quad \frac{L^{2}}{2} \sim \frac{8}{s}, \\
L & \sim \frac{4}{\sqrt{s}} .
\end{aligned}
$$

Hence

$$
\alpha=\frac{4}{\pi} \exp \left(\frac{-\pi^{2} \sqrt{s}}{24}\right)
$$

According to (3.2), we can take $M=C_{1} s^{3}$. On the other hand, by (3.1),

$$
|f(1)|=\left|a_{\Delta}(s, \cos (\theta)=1)\right|>\frac{C_{2}}{s^{5} \log (s)^{2}} .
$$

Therefore [using (3.6)]

$$
\begin{aligned}
m & =\sup _{|\cos (\theta)|<\frac{1}{2}} \mid a_{\Delta}(s, \cos (\theta) \mid \\
& \geq C_{3} s^{3}\left[C_{4} s^{8} \log (s)^{2}\right]^{-\frac{\pi}{4} \exp \left(\frac{\pi^{2} \sqrt{s}}{24}\right)} .
\end{aligned}
$$

This clearly implies similar lower bounds for $A_{s}(s, t)$ and $F(s, t)$.
This result is rather disappointing but, as a matter of principle, we see that it is not zero. For different values of $a$ we get the same qualitative behavior. For a nonsymmetric amplitude, like $\pi^{+} \pi^{-} \rightarrow \pi^{+} \pi^{-}$, we can get a lower bound for $-1 \leq \cos \theta \leq-a, 0 \leq a<1$. Spin complications can be overcome. Following Mahoux and Martin [13] we can take as the amplitude the sum of all diagonal helicity amplitudes, which, in both the $s$ channel and the $u$ channel, has the right positivity properties of the absorptive parts.

\section{APPENDIX A: LOWER BOUNDS FOR THE PARTICLE-PARTICLE AND PARTICLE-ANTIPARTICLE FORWARD SCATTERING AMPLITUDES}

In [3] a lower bound for a crossing-symmetric forward scattering amplitude was obtained. This is the case for, for instance, the $\pi_{0} p \rightarrow \pi_{0} p$ scattering amplitude. If the scattering amplitude is not crossing-symmetric, we can always symmetrize it, but then one gets a lower bound only on the average, say $\frac{1}{2}[A B \rightarrow A B+A \bar{B} \rightarrow A \bar{B}]$; we can only say that it applies to one of the amplitudes, but one does not know which one. Here, at the price of a very small weakening of the lower bound, we get lower bounds separately for $A B \rightarrow A B$ and $A \bar{B} \rightarrow A \bar{B}$.

We assume that the forward scattering amplitude satisfies a dispersion relation with a finite number of subtractions $N$. Above the right-hand cut $\operatorname{Im} F_{A B \rightarrow A B}(s)>0$. On the lefthand cut $\operatorname{Im} F_{A \bar{B} \rightarrow A \bar{B}}(u)>0$, with $u=2\left(M_{A}^{2}+M_{B}^{2}\right)-s$, which means that, above the left-hand cut, the imaginary part is negative.

First we study a function $G(z)=\overline{G(\bar{z})}$ with a positive imaginary part above both cuts, with a finite number of subtractions $N$. We may suppose $N$ even (otherwise we use $N+1)$. $\operatorname{Im} G(z)$ vanishes in an open interval containing 0 .

$$
G(z)=\sum_{n=0}^{N-1} c_{n} z^{n}+\frac{z^{N}}{\pi} \int_{-\infty}^{+\infty} \frac{\operatorname{Im} G\left(z^{\prime}\right) d z^{\prime}}{\left(z^{\prime}-z\right) z^{\prime N}},
$$

$\operatorname{Im} G(z) \geq 0$ for real $z$.

First we shall prove that $\frac{G(i y)}{y^{N}} \rightarrow 0$ when $y \rightarrow+\infty$.

$$
G(i y)=\sum_{n=0}^{N-1} c_{n}(i y)^{n}+\frac{(i y)^{N}}{\pi} \int_{-\infty}^{+\infty} \frac{\operatorname{Im} G\left(z^{\prime}\right) d z^{\prime}}{\left(z^{\prime}-i y\right) z^{\prime N}}
$$

$|G(i y)| \leq$ Polynomial of degree $N-1$

$$
\begin{aligned}
& +\frac{y^{N}}{\pi} \int_{-\infty}^{-M} \frac{\operatorname{Im} G\left(z^{\prime}\right) d z^{\prime}}{\left|z^{\prime}\right|^{N+1}} \\
& +\frac{y^{N}}{\pi} \int_{M}^{\infty} \frac{\operatorname{Im} G\left(z^{\prime}\right) d z^{\prime}}{z^{N+1}} \\
& +\frac{y^{N-1}}{\pi} \int_{-M}^{M} \frac{\operatorname{Im} G\left(z^{\prime}\right) d z^{\prime}}{\left|z^{\prime}\right|^{N}} .
\end{aligned}
$$




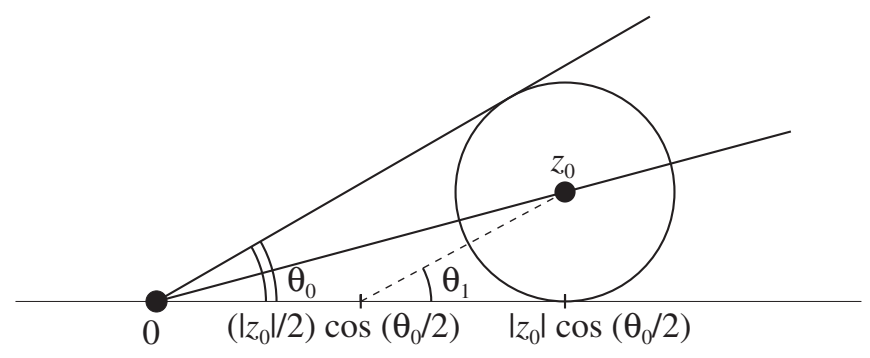

FIG. 1. The point $z_{0}$.

By taking $M$ large enough, we can make the first two terms less than $\varepsilon y^{N}$. The third term is bounded by $C y^{N-1}=\frac{1}{y} C y^{N}$. So, for $y \rightarrow \infty,\left|\frac{G(i y)}{y^{N}}\right| \leq 3 \varepsilon$; however, $\varepsilon$ can be taken arbitrarily small, so

$$
\lim _{y \rightarrow \infty}\left|\frac{G(i y)}{y^{N}}\right|=0
$$

Suppose we have, at the same time,

$$
\lim \left|\frac{G(z)}{z^{N}}\right| \rightarrow 0 \quad \text { for }|z| \rightarrow \infty, \quad \operatorname{Arg} z=\frac{\pi}{2},
$$

and

$$
G(z)|z|^{1+\alpha} \rightarrow 0 \quad \text { for }|z| \rightarrow \infty, \quad \operatorname{Arg} z=0 .
$$

Then construct $H$ :

$H(z)=G(z) \exp \left[(1+\alpha) \log z+\frac{i}{\pi}(N+1+\alpha)(\log z)^{2}\right]$.

Then $H(z)$ tends to 0 as $|z| \rightarrow \infty$ both for $\operatorname{Arg} z=0$ and $\operatorname{Arg} z=\frac{\pi}{2}$. By the Phragmén-Lindelöf theorem $H(z)$ tends to 0 as $|z| \rightarrow \infty$ for $0 \leq \operatorname{Arg} z \leq \frac{\pi}{2}$. Thus,

$$
\lim _{|z| \rightarrow \infty}|G(z)||z|^{\left(1+\alpha-\frac{2}{\pi} \theta(1+\alpha+N)\right)}=0, \quad \theta=\operatorname{Arg} z \in\left[0, \frac{\pi}{2}\right] .
$$

In particular,

$$
\lim _{|z| \rightarrow \infty}|G(z)||z|^{\left(1+\frac{\alpha}{2}\right)}=0 \quad \text { for } 0 \leq \theta \leq \theta_{0}=\frac{\pi}{4} \frac{\alpha}{1+\alpha+N} .
$$

In what follows we shall eliminate subtractions by differentiating $G N+1$ times. We need a bound on $\left(\frac{d}{d z}\right)^{N+1} G(z)$ somewhere in the angle $0<\theta<\theta_{0}$. We shall take $z_{0}=\left|z_{0}\right| e^{i \frac{\theta_{0}}{2}}$. The disk $\left|z-z_{0}\right|<\left|z_{0}\right| \sin \left(\theta_{0} / 2\right)$ is contained in the angular interval $0<\theta<\theta_{0}$ (see Fig. 1).
Using the Cauchy integral for $\left(\frac{d}{d z}\right)^{N+1} G(z)$ at $z=z_{0}$, we get

$$
\begin{aligned}
& \lim _{z_{0} \rightarrow \infty}\left[\left|z_{0}\right|\left(1-\sin \frac{\theta_{0}}{2}\right)\right]^{\left(1+\frac{\alpha}{2}\right)} \\
& \quad \times \frac{\left|z_{0} \sin \left(\frac{\theta_{0}}{2}\right)\right|^{N+1}}{(N+1) !}\left(\frac{d}{d z}\right)^{N+1} G\left(z_{0}\right)=0 .
\end{aligned}
$$

However, we can also estimate $\left(\frac{d}{d z}\right)^{N+1} G(z)$ from the dispersion relation

$$
\left(\frac{d}{d z}\right)^{N+1} G(z)=\frac{(N+1) !}{\pi} \int_{-\infty}^{\infty} \frac{\operatorname{Im} G\left(z^{\prime}\right) d z^{\prime}}{\left(z^{\prime}-z\right)^{N+2}} .
$$

We shall find a lower bound for the real part of this quantity. Here the positivity of $\operatorname{Im} G\left(z^{\prime}\right)$ in the integral is essential, and the evenness of $N$ is used.

We suppose $0<\alpha<1$. If $z^{\prime} \leq \frac{1}{2}\left|z_{0}\right| \cos \frac{\theta_{0}}{2}$ we have (see Fig. 1)

$$
\begin{aligned}
0<\operatorname{Arg}\left(z_{0}-z^{\prime}\right) & \leq \theta_{1}=\operatorname{Arg}\left(z_{0}-\frac{1}{2}\left|z_{0}\right| \cos \frac{\theta_{0}}{2}\right) \\
& <\theta_{0}=\frac{\pi}{4} \frac{\alpha}{1+\alpha+N},
\end{aligned}
$$

$$
0<\operatorname{Arg}\left(\left(z_{0}-z^{\prime}\right)^{N+2}\right)<\frac{\pi}{4} \frac{(N+2) \alpha}{1+\alpha+N}<\frac{\alpha \pi}{2} .
$$

Therefore

$$
\begin{aligned}
\operatorname{Re}\left(\frac{d}{d z}\right)^{N+1} G\left(z_{0}\right) \geq & \frac{(N+1) !}{\pi} \int_{-\infty}^{\frac{1}{2}\left|z_{0}\right| \cos \frac{\theta_{0}}{2}} \\
& \times \frac{\operatorname{Im} G\left(z^{\prime}\right) \cos (\alpha \pi / 2) d z^{\prime}}{\left|z^{\prime}-z_{0}\right|^{N+2}} \\
& -\frac{(N+1) !}{\pi} \int_{\frac{1}{2}\left|z_{0}\right| \cos \frac{\theta_{0}}{2}}^{\infty} \\
& \times \frac{1}{\left|z_{0} \sin \frac{\theta_{0}}{2}\right|^{N+2}} \operatorname{Im} G\left(z^{\prime}\right) d z^{\prime},
\end{aligned}
$$

but $\operatorname{Im} G\left(z^{\prime}\right)<C\left|z^{\prime}\right|^{-(1+\alpha)}$ from (A6); thus, the second term is less than $\left|z_{0}\right|^{-(N+2+\alpha)}$. The first term is larger than

$$
\frac{C}{\left|z_{0}+M\right|^{N+2}} \int_{-M}^{M} \operatorname{Im} G\left(z^{\prime}\right) d z^{\prime} .
$$

Thus, for $\left|z_{0}\right|$ large, the second term is negligible, but then (A15) contradicts (A10). The conclusion is that

$\lim _{z \rightarrow \infty} \sup |G(z)| z^{1+\alpha}>0, \quad \alpha>0$ arbitrarily small.

In fact, it reaches infinity. 


\section{Application to the scattering amplitude}

Note that $F(s)$ satisfies a dispersion relation with two cuts corresponding to the processes $A B \rightarrow A B$ and $A \bar{B} \rightarrow A \bar{B}$. Define $z=s-M_{A}^{2}-M_{B}^{2}$. We keep the notation $F(z)$. Since the imaginary part above the right-hand cut is positive and the imaginary part above the left-hand cut is negative, multiply $F(z)$ by $z$ :

$$
G(z)=z F(z) .
$$

Then the previous results apply, and we have separately

$$
\lim _{s \rightarrow+\infty} \sup |F(s)| s^{2+\alpha}>0,
$$

$\alpha>0$ arbitrarily small, for the reaction $A B \rightarrow A B$, and

$$
\lim _{u \rightarrow+\infty} \sup |F(u)| u^{2+\alpha}>0,
$$

for the reaction $A \bar{B} \rightarrow A \bar{B}$.

Notice that these results persist if we convolute $F$ with a positive function with compact support $w$ :

$$
F_{w}(s)=\int F\left(s^{\prime}\right) w\left(s-s^{\prime}\right) d s^{\prime} .
$$

\section{APPENDIX B: A PROBLEM OF ESTIMATION}

Let $f$ be a function holomorphic on the domain $D_{L}$ bounded by the ellipse $E_{L}$ with foci \pm 1 and semi-major axis $\operatorname{ch}(L), L>0$,

$$
\begin{gathered}
E_{L}=\{z: z=\cos (\theta+i L), \theta \in \mathbf{R}\}, \\
D_{L}=\{z: z=\cos (\theta+i y), \theta \in \mathbf{R}, y \in \mathbf{R},|y|<L\} .
\end{gathered}
$$

We suppose

$$
\begin{array}{ll}
|f(z)| \leq M & \text { for all } z \in D_{L}, \\
|f(z)| \leq m & \text { for all } z \in[-a, a],
\end{array}
$$

where $0<m<M$ and $a=\cos (b), 0<a<1,0<b<$ $\pi / 2$. We seek an upper bound for $|f(1)|$. General theorems (see e.g., pages 141-145 in [14]) assert the existence and uniqueness of a function $H$, harmonic on $D_{L} \backslash[-a, a]$ (i.e., $D_{L}$ minus a cut along the segment $\left.[-a, a]\right)$ and continuous at the boundary, and such that $H=1$ on $E_{L}$ and $H=0$ on $[-a, a]$. For every $z \in D_{L} \backslash[-a, a], 0<H(z)=H(-z)=$ $H(\bar{z})<1$ and

$$
\begin{aligned}
\log |f(z)| & \leq H(z) \log (M)+[1-H(z)] \log (m) \\
\forall z & \in D_{L} .
\end{aligned}
$$

Indeed $\log |f(z)|$ is subharmonic in $D_{L} \backslash[-a, a]$, and at the boundary it is majorized by the harmonic function which

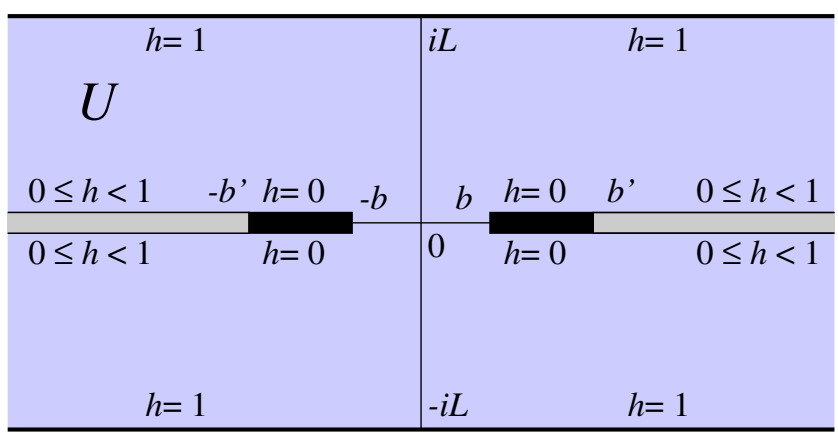

FIG. 2. The domain $U$ and boundary values for $h$.

appears on the rhs of (B3) (see e.g., pages 16-18 in [15] and page 132 in [14]). In particular,

$$
\log |f(1)| \leq H(1) \log (M)+[1-H(1)] \log (m) .
$$

It is possible to give an exact determination of $H$, but we give cruder upper and lower bounds for it, which describe more explicitly its behavior when $L$ tends to 0 . We use the notation

$\mathbf{C}_{+}=-\mathbf{C}_{-}=\{z \in \mathbf{C}: \operatorname{Im} z>0\}, \mathbf{D}=\{z \in \mathbf{C}:|z|<1\}$.

By a "conformal map" we always mean a holomorphic injective map.

\section{Upper and lower bounds for $\boldsymbol{H}$}

Let $h(z)=H(\cos (z))$. This function is harmonic and even, and it has period $\pi$ in the domain

$$
S_{L} \backslash \bigcup_{n \in \mathbf{Z}}(I+n \pi)
$$

$$
S_{L}=\{z:|\operatorname{Im} z|<L\},
$$

$$
I=\left[b, b^{\prime}\right], \quad b^{\prime}=\pi-b>\frac{\pi}{2}>b .
$$

Note that $-I=I-\pi$ and that $\bigcup_{n \in \mathbf{Z}}(I+n \pi)=$ $\cos ^{-1}([-a, a])$. In the domain (B6), $0<h(z)<1$. The function $h$ is continuous at the boundary of this domain and takes the value 1 on the edges of $S_{L}$, i.e., $\mathbf{R} \pm i L$, and the value 0 on the cuts $I+n \pi$. Note that $H(1)=h(0)$.

Let $U$ be the smaller domain consisting of the strip $S_{L}$ minus two cuts on $(-\infty,-b]$ and $[b,+\infty)$. Note that $h$ is harmonic in $U$ and continuous at its boundary (see Fig. 2).

We can conformally map $U$ onto the upper half-plane by a map $\psi_{2} \circ \psi_{1}$.

The map $\psi_{1}$ conformally maps the strip $S_{L}=\{z$ : $|\operatorname{Im} z|<L\}$ onto the cut plane $\mathbf{C}_{+} \cup \mathbf{C}_{-} \cup(-1,1)$ : 


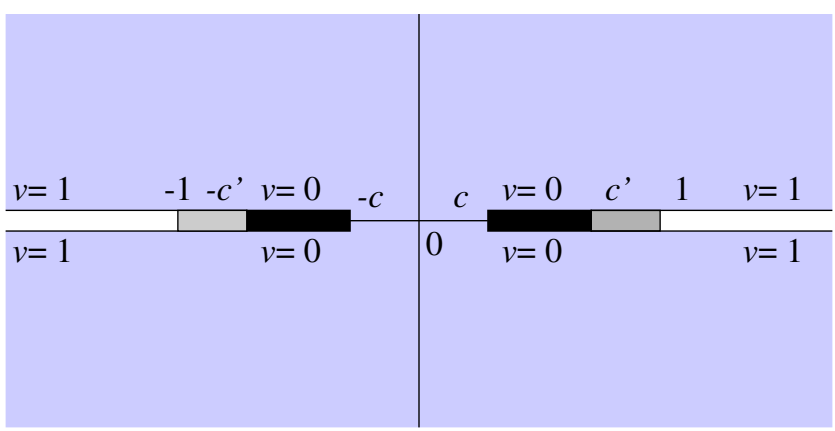

FIG. 3. Domain and boundary values for $v$.

$Z=\psi_{1}(z) \Leftrightarrow z=\frac{L}{\pi} \log \left(\frac{1+Z}{1-Z}\right), \quad Z=\operatorname{th}\left(\frac{\pi z}{2 L}\right)$.

It maps the cut strip $U$ pictured in Fig. 2 onto the cut plane $\mathbf{C}_{+} \cup \mathbf{C}_{-} \cup(-c, c)$, where

$c=\psi_{1}(b)=\operatorname{th}\left(\frac{\pi b}{2 L}\right), \quad c^{\prime}=\psi_{1}\left(b^{\prime}\right)=$ th $\left(\frac{\pi b^{\prime}}{2 L}\right)$.

Recall that $b^{\prime}=\pi-b>b$ so that $1>c^{\prime}>c$. Denoting $v(Z)=h\left(\psi_{1}^{-1}(Z)\right)$, the domain and boundary values for $v$ are pictured in Fig. 3. The points $Z= \pm 1$ are images of $z= \pm \infty$, so $v$ is not continuous there. It is continuous at all the other boundary points of the cut plane.

The map $\psi_{2}$ conformally maps the cut plane $\mathbf{C}_{+} \cup \mathbf{C}_{-} \cup$ $(-c, c)$ (pictured in Fig. 3) onto the upper half-plane $\mathbf{C}_{+}$.

$$
\begin{aligned}
& \zeta=\psi_{2}(Z) \Leftrightarrow Z=\frac{c}{2}\left(\zeta+\frac{1}{\zeta}\right), \\
& \zeta=\frac{Z}{c}+\sqrt{\left(\frac{Z}{c}\right)^{2}-1 .}
\end{aligned}
$$

In this formula the function $t \mapsto \sqrt{t^{2}-1}$ is defined to be holomorphic with a positive imaginary part in the cut plane $\mathbf{C}_{+} \cup \mathbf{C}_{-} \cup(-1,1)$. Hence $\psi_{2}(\bar{Z})=1 / \overline{\psi_{2}(Z)}$. If $t$ is real with $t>1$, then

$$
\begin{aligned}
& (t+i 0)+\sqrt{(t+i 0)^{2}-1}=t+\left|\sqrt{t^{2}-1}\right|+i 0 \\
& (t-i 0)+\sqrt{(t-i 0)^{2}-1}=t-\left|\sqrt{t^{2}-1}\right|+i 0 \\
& =\frac{1}{t+\left|\sqrt{t^{2}-1}\right|}+i 0
\end{aligned}
$$

The image $\psi_{2}\left(\mathbf{C}_{-}\right)$of the lower half-plane is $\mathbf{D} \cap \mathbf{C}_{+}$, $\psi_{2}(0)=i$, and $\psi_{2}(-i \infty)=0$. Let $A_{0}=\psi_{2}(1-i 0)$, $B_{0}=\psi_{2}(1+i 0), A=\psi_{2}\left(c^{\prime}-i 0\right), B=\psi_{2}\left(c^{\prime}+i 0\right)$, i.e.,

$$
\begin{aligned}
B_{0} & =\frac{1}{c}+\sqrt{\left(\frac{1}{c}\right)^{2}-1}=\frac{1}{A_{0}}, \\
B & =\frac{c^{\prime}}{c}+\sqrt{\left(\frac{c^{\prime}}{c}\right)^{2}-1}=\frac{1}{A}, \\
A_{0} & <A<1<B<B_{0} .
\end{aligned}
$$

Let

$$
u(\zeta)=v\left(\psi_{2}^{-1}(\zeta)\right)=h\left(\psi_{1}^{-1}\left(\psi_{2}^{-1}(\zeta)\right)\right) .
$$

In other words, $u$ is the result of transporting $h$ by the successive coordinate changes $z \rightarrow Z \rightarrow \zeta$. In particular, $u(i)=h(0)=H(1)$. The function $u$ is harmonic in the upper half-plane $\mathbf{C}_{+}$and $0<u(\zeta)<1$ for all $\zeta \in \mathbf{C}_{+}$. The function $1-u$ has the same properties and it is continuous at the real points except at 0 and $\pm A_{0}$ and $\pm B_{0}$. Its boundary values at other points are

$$
\begin{aligned}
1-u(t+i 0) & =1 \text { for } t \in[-B,-A] \cup[A, B], \\
1-u(t+i 0) & =0 \text { for } t \in \mathbf{R} \backslash\left(\left[-B_{0},-A_{0}\right] \cup\left[A_{0}, B_{0}\right] \cup\{0\}\right), \\
0 & <1-u(t+i 0) \leq 1 \\
\text { for } t & \in\left(-B_{0},-B\right) \cup\left(-A,-A_{0}\right) \cup\left(A_{0}, A\right) \cup\left(B, B_{0}\right) .
\end{aligned}
$$

If $x_{1}$ and $x_{2}$ are real with $x_{1}<x_{2}$, let

$$
\chi_{x_{1}, x_{2}}(\zeta)=\log \left(\frac{\zeta-x_{2}}{\zeta-x_{1}}\right)
$$

be defined as holomorphic in $\mathbf{C} \backslash\left[x_{1}, x_{2}\right]$, and mapping $\mathbf{C}_{+}$ (resp. $\mathbf{C}_{-}$) into itself. If $\zeta \in \mathbf{C}_{+}, \operatorname{Im} \chi_{x_{1}, x_{2}}(\zeta)$ is in $(0, \pi)$, and it is the angle under which the segment $\left(x_{1}, x_{2}\right)$ is seen from the point $\zeta$. It is a harmonic function in $\mathbf{C}_{+}$, continuous at all real points except $x_{1}$ and $x_{2}$, with boundary values equal to 0 outside of $\left[x_{1}, x_{2}\right]$, and to $\pi$ on $\left(x_{1}, x_{2}\right)$. It tends to 0 at infinity in the closed upper half-plane.

For $\zeta \in \mathbf{C}_{+}$, let $u_{+}(\zeta)$ and $u_{-}(\zeta)$ be defined by

$$
\begin{aligned}
& 1-u_{+}(\zeta)=\frac{1}{\pi} \operatorname{Im} \chi_{-B,-A}(\zeta)+\frac{1}{\pi} \operatorname{Im} \chi_{A, B}(\zeta), \\
& 1-u_{-}(\zeta)=\frac{1}{\pi} \operatorname{Im} \chi_{-B_{0},-A_{0}}(\zeta)+\frac{1}{\pi} \operatorname{Im} \chi_{A_{0}, B_{0}}(\zeta) .
\end{aligned}
$$

For $\zeta \in \mathbf{C}_{+}, 1-u_{ \pm}(\zeta) \in(0,1)$ since the angles under which $\left[-B_{0},-A_{0}\right]$ and $\left[A_{0}, B_{0}\right]$ are seen from $\zeta$ add up to less than $\pi$.

In particular, recalling that $A=1 / B$,

$$
\begin{aligned}
\operatorname{Im} \chi_{-B,-A}(i)= & \operatorname{Im} \chi_{A, B}(i)=\operatorname{Arctg}(B)-\operatorname{Arctg}(A) \\
= & 2 \operatorname{Arctg}(B)-\frac{\pi}{2}, \\
& 1-u_{+}(i)=\frac{4}{\pi} \operatorname{Arctg}(B)-1 .
\end{aligned}
$$




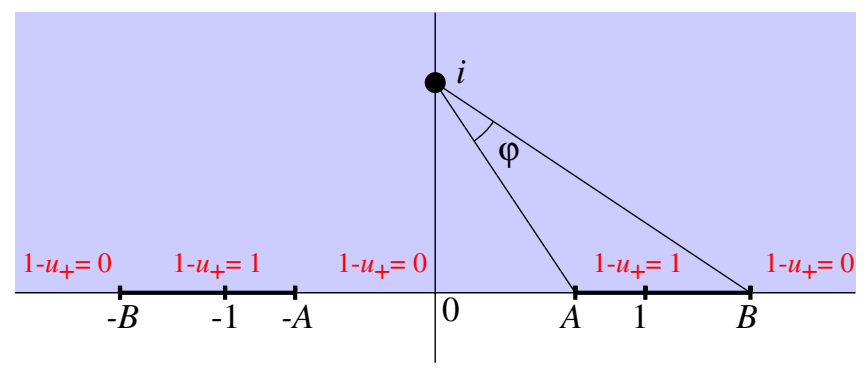

FIG. 4. Domain and boundary values for $1-u_{+} ; \varphi=$ $\operatorname{Im} \chi_{A, B}(i)$. The picture for $1-u_{-}$is the same with $A_{0}$ and $B_{0}$ instead of $A$ and $B$.

See Fig. 4. Similarly

$$
1-u_{-}(i)=\frac{4}{\pi} \operatorname{Arctg}\left(B_{0}\right)-1 .
$$

The boundary values of $1-u_{+}$are (see Fig. 4)

$$
\begin{gathered}
1-u_{+}(t+i 0)=1 \text { for } t \in(-B,-A) \cup(A, B), \\
1-u_{+}(t+i 0)=0 \text { for } t \notin[-B,-A] \cup[A, B] .
\end{gathered}
$$

Those of $1-u_{-}$are

$1-u_{-}(t+i 0)=1 \quad$ for $t \in\left(-B_{0},-A_{0}\right) \cup\left(A_{0}, B_{0}\right)$,

$1-u_{-}(t+i 0)=0$ for $t \notin\left[-B_{0},-A_{0}\right] \cup\left[A_{0}, B_{0}\right]$.

Thus, except at a finite set of real points, we have

$$
\begin{aligned}
1-u_{+}(\zeta+i 0) & \leq 1-u(\zeta+i 0) \leq 1-u_{-}(\zeta+i 0), \\
\zeta & \in \mathbf{R} .
\end{aligned}
$$

In spite of the exceptional points we can still apply the maximum (or minimum) principle in the form of the following lemma

Lemma B. 1.- Let $g$ be a continuous function on the closed upper half-plane with the exception of a finite set $\mathcal{F}$ of real points. We suppose that $|g(\zeta)|<C<\infty$ and $g$ is harmonic in $\mathbf{C}_{+}$, and $g(\zeta) \geq 0$ for all $\zeta \in \mathbf{R} \backslash \mathcal{F}$. Then $g(\zeta) \geq 0$ for all $\zeta \in \mathbf{C}_{+}$. If we assume that $g(\zeta)=$ 0 for all $\zeta \in \mathbf{R} \backslash \mathcal{F}$, then $g(\zeta)=0$ for all $\zeta \in \mathbf{C}_{+}$.

(The boundedness of $|g|$ is essential as the example of $\zeta \mapsto-\operatorname{Im} \zeta$ shows). To prove this lemma, choose a fixed $\zeta_{0} \in \mathbf{C}_{+}$. The function $\varphi(\zeta)=i\left(\zeta-\zeta_{0}\right) /\left(\zeta-\bar{\zeta}_{0}\right)$ maps $\mathbf{C}_{+}$onto $\mathbf{D}$, the closed upper half-plane onto $\overline{\mathbf{D}} \backslash\{i\}$, and $\varphi\left(\zeta_{0}\right)=0$. The function $G(z)=g\left(\varphi^{-1}(z)\right)$ is harmonic in $\mathbf{D}$ and continuous on $\overline{\mathbf{D}} \backslash \mathcal{F}_{1}$ where $\mathcal{F}_{1}$ is a finite subset of the unit circle. Wherever defined, $|G(z)| \leq C$. Let $\mathcal{F}_{2}=\left\{\theta \in[0,2 \pi]: e^{i \theta} \in \mathcal{F}_{1}\right\}$. For sufficiently small $\kappa \in$ $(0,1)$ there is a compact subset $E_{\kappa}$ of $[0,2 \pi]$ whose
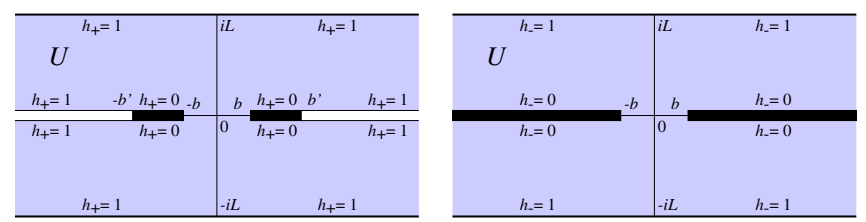

FIG. 5. Domain and boundary values for $h_{+}$(left) and $h_{-}$ (right).

complement contains $\mathcal{F}_{2}$ and has measure $\leq 2 \pi \kappa$, and an $r_{\kappa} \in(0,1)$ such that $\left|G\left(e^{i \theta}\right)-G\left(r_{\kappa} e^{i \theta}\right)\right|<\kappa$ for all $\theta \in E_{\kappa}$. For $\theta \in E_{\kappa}, G\left(e^{i \theta}\right) \geq 0$. Therefore

$$
\begin{aligned}
G(0) & =\int_{0}^{2 \pi} G\left(r_{\kappa} e^{i \theta}\right) \frac{d \theta}{2 \pi} \geq \int_{E_{\kappa}} G\left(r_{\kappa} e^{i \theta}\right) \frac{d \theta}{2 \pi}-C \kappa \\
& \geq \int_{E_{\kappa}} G\left(e^{i \theta}\right) \frac{d \theta}{2 \pi}-\kappa-C \kappa \geq-(C+1) \kappa .
\end{aligned}
$$

Letting $\kappa$ tend to 0 we get $G(0)=g\left(\zeta_{0}\right) \geq 0$. If we assume that $g(\zeta)=0$ for all $\zeta \in \mathbf{R} \backslash \mathcal{F}$, then also $-g(\zeta) \geq 0$ for all $\zeta \in \mathbf{C}_{+}$; hence $g(\zeta)=0$ for all $\zeta \in \mathbf{C}_{+}$.

Applying this to $g=u_{+}-u$ and to $g=u-u_{-}$, we find that $u_{-}(\zeta) \leq u(\zeta) \leq u_{+}(\zeta)$ for all $\zeta \in \mathbf{C}_{+}$. In particular, $1-u(\zeta)$ tends to 0 if $\zeta$ tends to 0 or infinity in the closed upper half-plane. In fact, the maximum principle implies that the inequalities are strict, i.e.,

$$
u_{-}(\zeta)<u(\zeta)<u_{+}(\zeta) \quad \forall \zeta \in \mathbf{C}_{+} .
$$

Hence

$$
\begin{aligned}
u_{-}(i) & =2-\frac{4}{\pi} \operatorname{Arctg}\left(B_{0}\right)<u(i)=H(1)<u_{+}(i) \\
& =2-\frac{4}{\pi} \operatorname{Arctg}(B) .
\end{aligned}
$$

To study the behavior of $u_{ \pm}(i)$ as $L \rightarrow 0$ we recall that, for real $z \geq 0$,

$$
\begin{gathered}
0 \leq \frac{d}{d z} \operatorname{Arctg}(1+z) \leq \frac{1}{2}, \\
-\frac{z^{2}}{4} \leq \operatorname{Arctg}(1+z)-\frac{\pi}{4}-\frac{z}{2} \leq 0 .
\end{gathered}
$$

We denote

$$
x=\exp \left(\frac{-\pi b}{L}\right), \quad x^{\prime}=\exp \left(\frac{-\pi b^{\prime}}{L}\right),
$$$$
b^{\prime}=\pi-b>b, \quad x^{\prime}<x .
$$

Note that $x^{\prime} / x \rightarrow 0$ as $L \rightarrow 0$. With this notation

$$
\begin{aligned}
\frac{1}{c} & =\frac{1+x}{1-x}, \quad c^{\prime}=\frac{1-x^{\prime}}{1+x^{\prime}}, \quad \sqrt{\left(\frac{1}{c}\right)^{2}-1}=\frac{2 \sqrt{x}}{(1-x)}, \\
B_{0} & =\frac{1}{c}+\sqrt{\left(\frac{1}{c}\right)^{2}-1}=\frac{1+\sqrt{x}}{1-\sqrt{x}}=1+\frac{2 \sqrt{x}}{1-\sqrt{x}} .
\end{aligned}
$$


Applying (B31) with $z=2 t /(1-t), t=\sqrt{x}$ gives

Hence

$$
\left|u_{-}(i)-1+\frac{4 \sqrt{x}}{\pi}\right| \leq \frac{4 x}{\pi(1-\sqrt{x})^{2}}, \quad x=\exp \left(\frac{-\pi b}{L}\right) .
$$

Further

$$
\begin{aligned}
& \frac{1}{c}-\frac{c^{\prime}}{c}=\frac{2 x^{\prime}}{c\left(1+x^{\prime}\right)}<\frac{2 x^{\prime}}{c}, \\
& \left(\frac{1}{c}\right)^{2}-\left(\frac{c^{\prime}}{c}\right)^{2}=\frac{4 x^{\prime}}{c^{2}\left(1+x^{\prime}\right)^{2}}<\frac{4 x^{\prime}}{c^{2}}, \\
& \sqrt{\left(\frac{1}{c}\right)^{2}-1}-\sqrt{\left(\frac{c^{\prime}}{c}\right)^{2}-1}<\frac{\frac{4 x^{\prime}}{c^{2}}}{\sqrt{\left(\frac{1}{c}\right)^{2}-1}}=\frac{2 x^{\prime}(1+x)}{c \sqrt{x}}, \\
& B_{0}-B<\frac{2 x^{\prime}}{c}\left[1+\frac{1+x}{\sqrt{x}}\right]<2 \sqrt{x^{\prime}}[1+\sqrt{x}+x]\left(\frac{1+x}{1-x}\right), \\
& \operatorname{Arctg}\left(B_{0}\right)-\operatorname{Arctg}(B)<\sqrt{x^{\prime}}[1+\sqrt{x}+x]\left(\frac{1+x}{1-x}\right) .
\end{aligned}
$$

Hence

$$
\begin{aligned}
& H(1)-u_{-}(i) \leq u_{+}(i)-u_{-}(i) \\
& \quad<\frac{4 \sqrt{x^{\prime}}}{\pi}[1+\sqrt{x}+x]\left(\frac{1+x}{1-x}\right) \\
& =\frac{4 \sqrt{x^{\prime}}}{\pi}(1+O(\sqrt{x})) .
\end{aligned}
$$

This gives

$$
u_{ \pm}(i)=1-\frac{4}{\pi} \exp \left(\frac{-\pi b}{2 L}\right)+\mathrm{o}\left(\exp \left(\frac{-\pi b}{2 L}\right)\right)(L \rightarrow 0),
$$

$$
H(1)=1-\frac{4}{\pi} \exp \left(\frac{-\pi b}{2 L}\right)+\mathrm{o}\left(\exp \left(\frac{-\pi b}{2 L}\right)\right)(L \rightarrow 0) .
$$

Bounds on the error terms are supplied by the preceding inequalities. Thus, although some information is lost if $H(1)$ is replaced by its upper bound $u_{+}(i)$, this becomes unimportant for very small $L$.

We also note that if we define $h_{ \pm}(z)=u_{ \pm}\left(\psi_{2}\left(\psi_{1}(z)\right)\right)$ (see Fig. 5),

$$
h_{-}(z)<h(z)<h_{+}(z) \quad \forall z \in U .
$$

The bounds obtained in this section are not useful at large $L$. In fact, when $L \rightarrow \infty,\left(c^{\prime} / c\right) \rightarrow\left(b^{\prime} / b\right)$; thus, $u_{+}(i)$ tends to a nonzero limit, while it can be shown that $H(1)$ tends to 0.
[1] F. A. Cerulus and A. Martin, A lower bound for large-angle elastic scattering at high energies. Phys. Lett. 8, 80 (1964).

[2] S. Mandelstam, Determination of the pion-nucleon scattering amplitude from dispersion relations and unitarity. General theory, Phys. Rev. 112, 1344 (1958); Analytic properties of transition amplitudes in perturbation theory, Phys. Rev. 115, 1741 (1959).

[3] Y. S. Jin and A. Martin, Connection between the asymptotic behavior and the sign of the discontinuity in one-dimensional dispersion relations, Phys. Rev. 135, B1369 (1964).

[4] W. F. Baker et al., E. W. Jenkins, A. L. Read, G. Cocconi, V. T. Cocconi, and J. Orear, Elastic $p-p$ Cross Sections at High-Momentum Transfers, Phys. Rev. Lett. 9, 221 (1962); W. F. Baker, E. W. Jenkins, A. L. Read, G. Cocconi, V. T. Cocconi, A. D. Krisch, J. Orear, R. Rubinstein, D. B. Scarl, and B. T. Ulrich, Large Angle $p-p$ Elastic Scattering at 30 bev, Phys. Rev. Lett. 12, 132 (1964).
[5] G. Veneziano, Construction of a crossing-symmetric, Regge behaved amplitude for linearly rising trajectories, Nuovo Cimento A 57, 190 (1968).

[6] A. Martin, Extension of the axiomatic analyticity domain of scattering amplitudes by unitarity. 1, Nuovo Cimento A $\mathbf{4 2}$, 930 (1966).

[7] H. Cornille, Forward lower bounds at high energy, Nuovo Cimento A 4, 549 (1971).

[8] A. Martin, An absolute upper bound on the pion-pion scattering amplitude, Stanford preprint ITP.1;1( 1964) and Qualitative and quantitative consequences of analyticity and unitarity, Proceedings of High Energy Physics and Elementary Particles, Trieste (IAEA, Vienna, 1965), p. 155.

[9] L. Lukaszuk and A. Martin, Absolute upper bounds for $\pi \pi$ scattering, Nuovo Cimento A 52, 122 (1967).

[10] C. Lopez and G. Mennessier, A new absolute bound on the $\pi^{0} \pi^{0}$ S-wave scattering length, Phys. Lett. 58B, 437 (1975); 
B. Bonnier, C. Lopez, and G. Mennessier, Improved absolute bounds on the $\pi^{0} \pi^{0}$ amplitude, Phys. Lett. 60B, 63 (1975); C. Lopez and G. Mennessier, Bounds on the $\pi^{0} \pi^{0}$ amplitude, Nucl. Phys. B118, 426 (1977).

[11] A. K. Common, Froissart bounds with no arbitrary constants, Nuovo Cimento A 69, 115 (1970).

[12] F. J. Yndurain, Absolute bound on cross-sections at all energies and without unknown constants, Phys. Lett. 31B, 368 (1970).
[13] G. Mahoux and A. Martin, Extension of axiomatic analyticity properties for particles with spin, and proof of superconvergence relations, Phys. Rev. 174, 2140 (1968).

[14] A. F. Beardon, A Primer on Riemann Surfaces, London Mathematical Society Lecture Note Series 78 (Cambridge University Press, Cambridge, England, 1984).

[15] L. Hörmander, An Introduction to Complex Analysis in Several Variables, 2nd ed. (North Holland, Amsterdam, 1973). 